\title{
EVOLVABLE ASSEMBLY SYSTEMS: FROM EVALUATION TO APPLICATION
}

\author{
Mauro Onori, Daniel Semere \\ $K T H$, \\ Stockholm, Sweden \\ \{onori,dte\}@ipp.kth.se \\ José Barata \\ UNINOVA / UNL, \\ Quinta da Torre, 2829-516 Caparica, Portugal \\ $\{l d r, j a b, p c m\} @ u n i n o v a . p t$
}

\begin{abstract}
Presented in 2002, and applied within the EUPASS and A3 projects, Evolvable Assembly Systems proposes a novel way of applying assembly systems in industry. The essence of EAS resides not only in the ability of system components to adapt to the changing conditions of operation, but also to assist in the evolution of these components in time such that processes may become more robust. The main features of Evolvable systems include distributed control, a modularized, intelligent and open architecture, and a comprehensive and multi dimensional methodological support that comprises the reference architecture. The work has been, and is being, implemented through large European research projects. Evolvability being a system concept, it is envisaged to address every aspect of an assembly system throughout its life cycle, i.e., design and development, operation and evolution. Furthermore, integration of legacy subsystems and modules have been addressed in the methodology. This article will present the latest developments, applications and conclusions drawn to date.
\end{abstract}

Key words: Evolvability, System Architecture, emergence, modular

\section{INTRODUCTION}

According to the Observatory of European SMEs [1], 93\% of all employees in Europe operate within companies with less than 10 workers/employees, and over 120 million people are directly employed in the European SME sector. Being the real giants of the European economies, their particular needs will need to be highlighted. Furthermore, it must be noted that due to the fact that manufacturing is becoming ever more customised, and the product lifecycles continue to decrease, the underlying needs of manufacturing become identical for both small and large enterprise. The bottom line is that almost $31 \%$ of the workforce in Europe is currently employed in the manufacturing industry, which means roughly 34 million employees or 1550 million $€$ in value adding activities.

The addition of the service sectors directly dependent on this manufacturing industry magnifies the figure significantly, and one is still not including the major European corporations!

According to the results attained by many roadmaps ([2],[3],[4],[5],[6]), one of the most important objectives to be met by European industry is sustainability, which is multifaceted: 
- the solutions need to be sustainable from an economical point of view as the companies need not only acquire the manufacturing technology, but also maintain it.

- $\quad$ there are ecological aspects linked to sustainability [7]: minimise use of resources $\&$ materials, waste disposal, pollution, etc.

- social aspects: as the technologies in question need to support and sustain the societies and economies being affected by them.

The obvious conclusion is that future manufacturing solutions will have to deal with very complex scenarios.

Evolvable Assembly Systems ([8], [9]) represents one of the paradigms proposed as an opportunity to solve such threats. It has, to date, resulted in several demonstrators and offered methodologies and architectures in support. This article will summarise the main achievements, but it is relevant to recall the reader that EAS is mainly a new paradigm and not a finalised, ready-to-use solution. This is of some importance as reconfigurability and flexibility have, after more than two decades of efforts, not produced any commercially successful results. Hence the need to explore new approaches.

\section{BACKGROUND APPROACHES}

The major problems incurred by companies dealing with assembly all relate to uncertainty. First of all, it is very difficult for companies to predict the type and range of products that will have to be developed. The second uncertainty regards the production volumes and lifespans reached by these future products.

For these reasons, in the process of development of a new product (or even a variant), companies need to optimise:

- the cost

- the Time-to-Market/ Time-to-Volume

In the case of a product-specific traditional assembly system, we have high initial costs and long assembly system development time, thus shortening the actual production lead times. Furthermore, each time a new product is required, investments tend to rise dramatically since the assembly system has to be adapted or exchanged.

The overwhelming reaction to these problems has been to attempt to develop extremely flexible assembly machines. In the late eighties and early nineties, the general trend in precision assembly was to develop Flexible Assembly Systems (FAS) and Flexible Automatic Assembly (FAA) cells (or Hyper Flexible Automatic Assembly, going a bit further with stepwise automation, standard assembly machines and sub-batch principle).

The goal was to have general flexibility, but the actual assembly processes were not studied in depth, therefore resulting in unstable / non-robust or badly adapted solutions. They were fairly adequate to many different product types, but failed to be very performing in any domain. The high cost of such installations was another heavy problem, especially for smaller companies. Flexibility, instead of the actual assembly process, has been the core issue of most of these developments. As shown in the Figure 1, the lower a component is positioned in the hierarchical structure, the more flexibility is necessary to ensure a certain flexibility to the whole system; this means even a system with low flexibility needs a very flexible control: this may imply that FAA solutions also failed because they never provided flexible/agile control systems! 


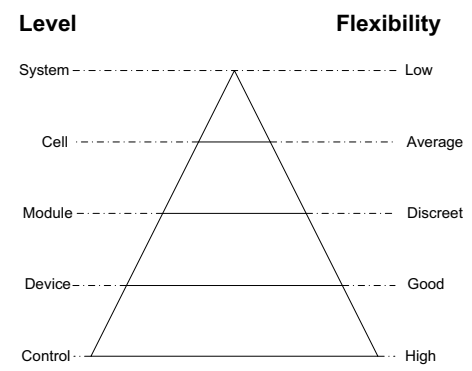

Figure 1. Possible levels of flexibility according to the hierarchical structure containing he component

The next attempt at finding a solution was Re-configurable Assembly Systems (RAS). In this case the focus was on the principle that innovative product design must not be limited by assembly process constraints.

In RMS, the Assembly System design starts from the "New Product Requirements". The product to be assembled is analysed in order to find all the "Assembly System Requirements", which means that the driver of the whole process is the Product. In such an approach there are no links between the Product Design process and the successive steps: it leaves the maximum freedom to the designers, but this is not always the right strategy for the company success.

\subsection{EAS}

Basically, the real objectives should not have been flexibility or reconfigurability. These are characteristics, not objectives. The real objective is system adaptability, which EAS targets with the following four points:

1. Optimised functionality: the assembly equipment is kept as simple as possible by deriving small, dedicated, process-oriented modules. These may be interconnected to form cells or systems.

2. Optimised orchestration: the control system needs to be the most agile aspects. This is achieved by adopting a multi-agent based, distributed control approach with embeded controllers.

3. Adaptability: the modularity allows for stepwise upgradeability and economoic flexibility (it is cheaper \& simpler to change a module than modify a system). The actual system may also adapt to minor changes via its control system, which, being skill-based, allows for emergent behaviour to be exploited.

4. Robustness: the equipment is dedicated, small, and includes an own processor. Some modules (robots) may even be reconfigurable. The control system is goaloriented, and the system is process-oriented. This results in a dedicated system based on an adatable concept with advanced interfaces.

Fundamentally, EAS suggests that true agility/flexibility can only be achieved if the lowest building blocks of a system are those that exhibit the highest rate of adaptability/evolvability. As the clustering of components increases in complexity, so does the agility/flexibility decrease. Hence, in order to build truly agile systems, one must begin by considering the control architecture.

According to the EAS Paradigm, each system should consist of several skill-based and process-oriented units: these elements should be very task-specific in order to accomplish only a simple action. It is possible, in fact, to consider every complex task as the union of 
several simple actions. Therefore each "shared element" can be used to accomplish the same action in different assembly cycles or in different product generations. Obviously, due to its modularity and unit-by-unit development, such a system is accessible even for small-medium sized companies that can spread the investment over a given timespan. Moreover, as will be detailed in Chapter 3, that the EAS process-oriented approach to the Ontology allows to define the Assembly System requirements for a whole Class of Products instead of a single product: the defined assembly processes are common to an entire set of products.

A fundamental condition is the plugability of all components.

One of the most important consequences of this approach relates to its control system: modules are agents, knowing their proper capabilities and possible forms of cooperation with other agents. The principles of emergence can be applied, working with module skills and forming higher capabilities out of it.

Therefore, when a system is created according to the EAS principles, the resulting capability of the sum of the modules will not be so easily predicted. When a multitude of small entities is brought together, new and unexpected capabilities surface from such coalitions [10]: this is called Emergent Behaviour. Of course the lower the level of the device considered, the higher is the emergent behaviour (fine granularity $=$ high emergence).

Evolvable Assembly Systems (EAS) exhibits the following characteristics:

- It is a fully "reconfigurable" system platform that exhibits an emergent behaviour.

- Mechatronically integratable assembly units.

- The reconfigurable system has to be composed of process-oriented components (gives granularity of emergent behaviour).

- A system that can automatically determine its functionality based on the components' skills.

- The change in paradigm is, partly, that we no longer invest in the programming \& coding, but, rather, in how to establish and exploit relations.

- Maintenance, documentation and the ability to store information in support of operational stability.

\section{METHODOLOGY}

The EAS Methodology provides the references architecture, enablers, and modelling formalisms. In the following section brief description of the methodology is given.

\subsection{Reference Architecture, RA}

The EAS Reference Architecture (EAS_RA) describes the essential features of an Evolvable System which means the reference architecture specifies the necessary features that a system should have to be an evolvable system.

The reference architecture is composed of three main elements: Principles, Technical Positions and Templates.

Principles : EAS has two fundamental principles which lay foundation and guide the development processs of an evolvable system. These principles can be considered as description of the core ideas of the evolvable system paradigm. 
Principle 1: the most innovative product design can only be achieved if no assembly process constraints are posed. The ensuing, fully independent, process selection procedure may then result in an optimal assembly system methodology.

Principle 2: Systems under a dynamic condition need to be evolvable, i.e., they need to have an inherent capability of evolution to address the new or changing set of requirements.

These principles have been described in detail in [11]:

Technical Positions: EAS design and implementation decisions and objectives set at a technical / technological level that describes the ontology, exploited protocols, standards or specifications for use with each major architectural component.

Templates and Partial Models: Reusable diagrams, graphs, objectives and knowledge and rules that address the distribution of system functions and how they relate topologically. Templates use models to show relationships and between components as specified by the Technical Positions and pertinent knowledge units.

\subsection{Modeling Formalisms}

Modelling formalisms refers to the ontology and the graphical tools used to build models in the reference architecture and the enabling models as described in section 3.3.

The EAS formalised concepts (ontology) and definitions are represented using a set of descriptive tools such as:

- Definitions of the most important concepts: module, process, product, eas module, skills, eas assembly system (which is a composition of modules), etc

- Diagrams (UML, etc) where the interactions between the concepts defined are shown. This enables to show how the EAS architecture generates assembly systems. The interaction may show the global system behaviour

- Formalisation of concepts

The domain ontology indeed captures the concepts in the system with their specifications (consensual semantic) i.e., what the concepts are and how they are related to each other in the domain. However, it does not capture the logic behind the relationships and the how's in the synthesis and functionality of the system.

\subsection{Enablers}

This part of the methodology provides the necessary models, tools and methods for the development and evolution of an evolvable system.

The enabling models include: the development process model, the business model and the knowledge model. These models are constructed using the formalisms described above and most notably the EAS ontology

The figure below depicts the first proposed EAS design methodology using IDEF0. The is a simplified high level activity model showing the main activities in the development process and their input, output, control and mechanisms needed to generate or modify instances of the architecture. 


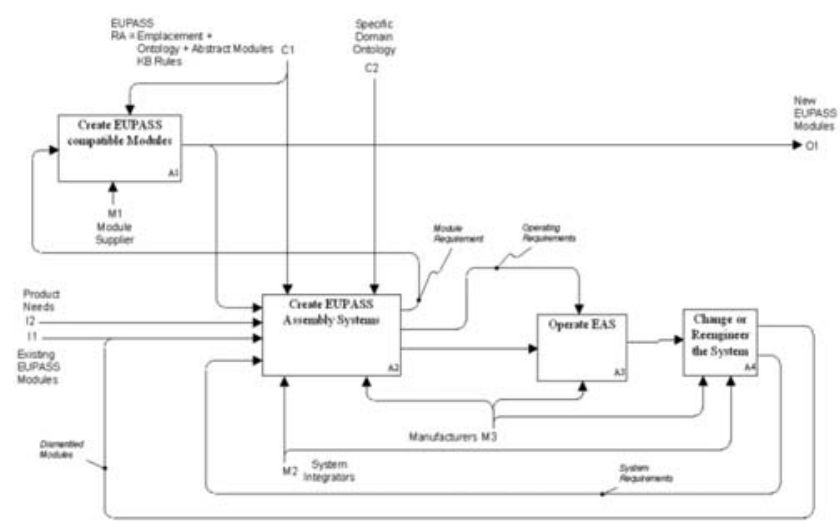

Figure 2. The simplified EAS Methodology

The traditional top-down system design is feasible only in cases where the emergent behaviour is fully describable; (Ueda, 2001). If emergent behaviour has to be investigated even at design and development stages, then a heterarchical or a network approach are the options.

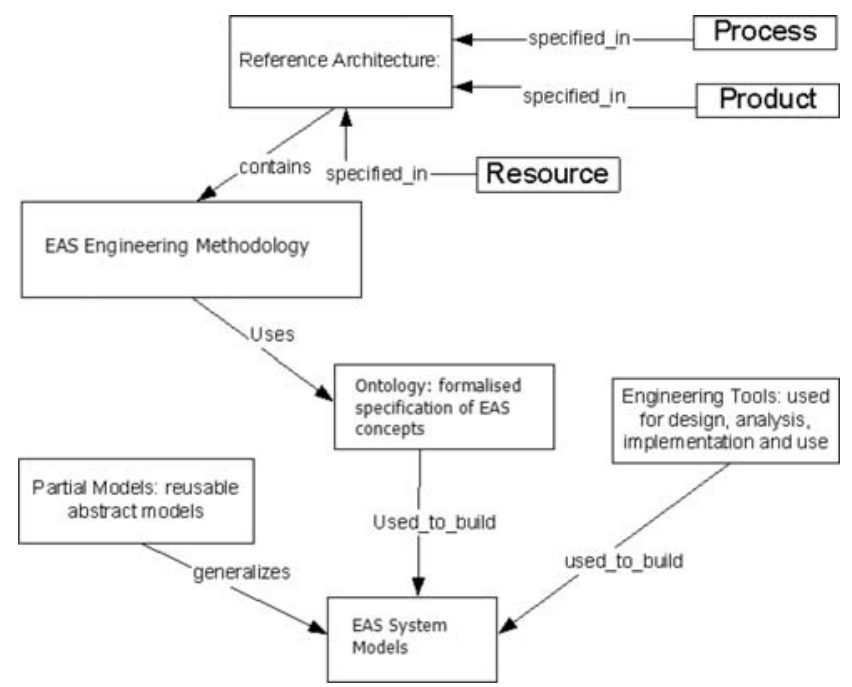

Figure 3. Basic Aspects of the EAS Reference Architecture

Concepts that are formalised in the EAS Ontology are used to capture the stakeholders understanding of their own domain. The EAS knowledge model is thus a structured and formalised collection of such knowledge capturing representations of the domains. The main objective of the EAS knowledge model is to provide an environment that supports the development and operation of evolvable systems. 


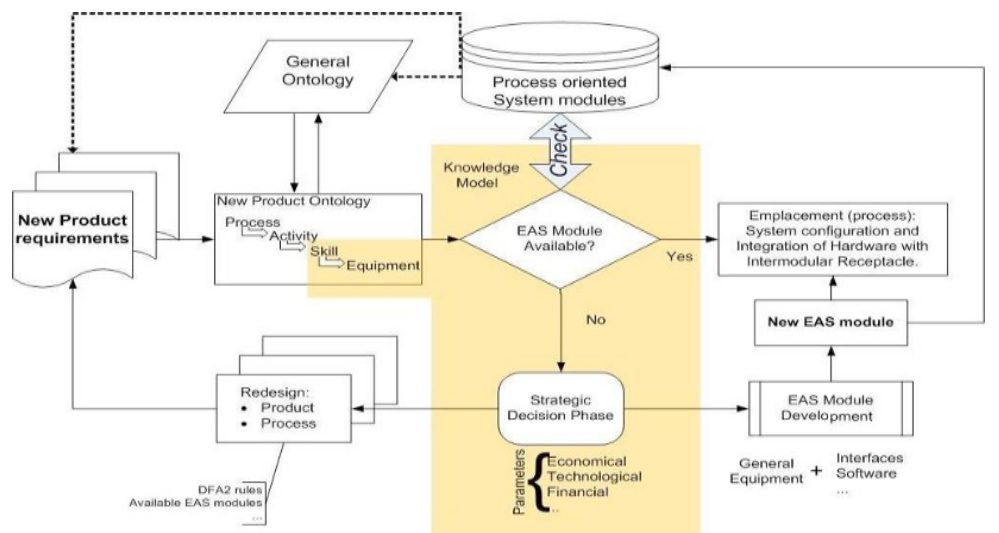

Figure 4. The preliminary EAS approach

The domain knowledge captured using the EAS ontology and the EAS knowledge templates are the two entities used to develop the knowledge model. The knowledge models are used among other things how each module in a system should address for a new set of conditions.

The elements in the EAS knowledge model consist of the following knowledge domains:

1. The enterprise knowledge domain - globalizes knowledge of the system and represents the business, organizational and global knowledge models captured. Enterprise knowledge enables environment recognition and maintenance of organizational associations.

2. The product knowledge domain - captures the knowledge related to product specification and design to assembly tasks.

3. The execution knowledge domain: capturing knowledge elements related to communications, planning and scheduling

The learning knowledge domain: containing knowledge elements that are used to incorporate case based reasoning.

The EAS RA is ultimately viewed from different perspectives addressing the different concerns of the stakeholders. The stakeholders include:

- those who build the system (structure and communication views),

- those who use it (functional view),

- those who are concerned with control (control view).

The architecture should, therefore, address the concerns of every stakeholder, which gives rise to a multiple set of viewpoints concerning the requirements, expected outputs and controlling mechanisms. These may come to include:

- Functional Views, which address the concerns of the users of the system, and describe the functionality of the system, the process flows, quality, logistical issues.

- Communication Views: which address the concerns of how the data and information is to be represented, interpreted, recorded and transmitted.

- Control views: which address the concerns of the control system.

- Structure Views, which address the concerns of the system integrators, and maintenance.

These represent the background work that needs to be carried out in order to develop and establish an adequate EAS_RA. The actual implementation of this architecture, when 
considering the EAS modules and how they are to be integrated and formed into a system, will also be subject to a set of "views".

\subsection{Intermodular Receptacle}

One of the most important aspects of developing an Evolvable Assembly system resides in the advanced interfacing requirements.

Every piece of equipment has its properties. They enable the equipment to perform or to assist at technical operations leading to the process goal. On the other side every piece of equipment has constraints on several levels. Moreover aspects like lifetime or maintenance cycles have to be taken into account. The design of assembly systems requires the awareness of both the abilities as well as the constraints. Automating the design process requires therefore a structured knowledge base including the equipments properties, comprehensive for operators or designers as well as for software services. If the technical properties were static and no runtime access to the properties was necessary it would make sense to just save everything in one large database. But modern mechatronic systems require fast and reliable data exchange. Furthermore regarding that the development of assembly systems is leading to more distributed systems, a knowledge base included in the equipment itself should be preferred. This equipment knowledge base can be regarded as a multi-level interface definition. It could be described completely with the contact and channel method, which actually is nothing else than an interface description, or with other more differentiated methods.

EAS is an approach to systemize the design of products and their assembly systems. The reasons mentioned above impose the inclusion of distributed knowledge. The optimisation goal of EAS is adaptability. To assure maximum adaptability, the knowledge bases have to be put at the lowest level available; i.e., at module level.

The module choice, in build time and in runtime, depends on the module's ability to perform skills and constraints imposed by general properties. A skill and its inherent information requires a structure and a place to store. To fit into yet proposed control concepts an implementation into the modules is also to be aimed at. Aiming at agility \& adaptability requires that as many components as possible fit together. This imposes standard interfaces, covering all relevant mechatronic aspects. Minimizing the amount of used interface types leads to a higher plugability and, therefore, agility on system design. In order to allow system designers to create new modules, by modifying legacy equipment or by developing something new, the interfaces have to be specified. This way the designers know how to develop a module fitting into the standard. The specification should also describe approaches and show examples.

The here described multi-level interface specification, including inherent module knowledge in form of a template and enabling the module to communicate its abilities with other system modules in form of skills, is called "Intermodular Receptacle"-see Figure 5 .

The development of the intermodular receptacle cammot be detailed at present due to pending patent applications, but these advanced interface devices will have embedded processing power. This will allow assembly modules to communicate between each other and attain self-configuration capability. 


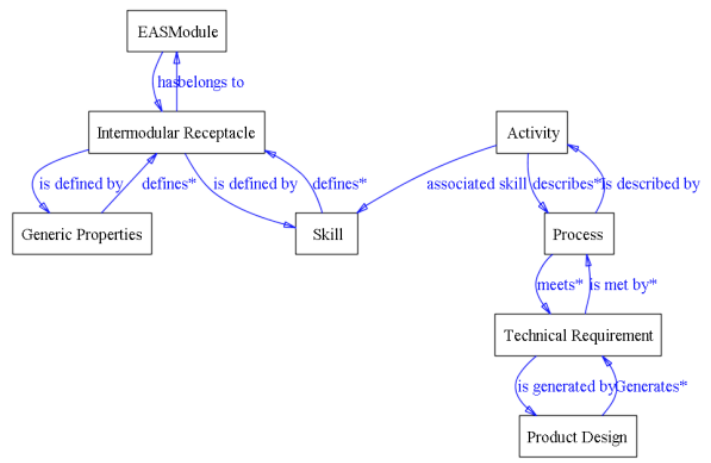

Figure 5. Ontological description of the Intermodular Receptacle

\section{APPLICATIONS}

Initial evaluations were carried out in the test cell shown below (Figure 6a). More industrially viable evaluations are currently being deployed within a new system being developed at KTH (Figure 6b).
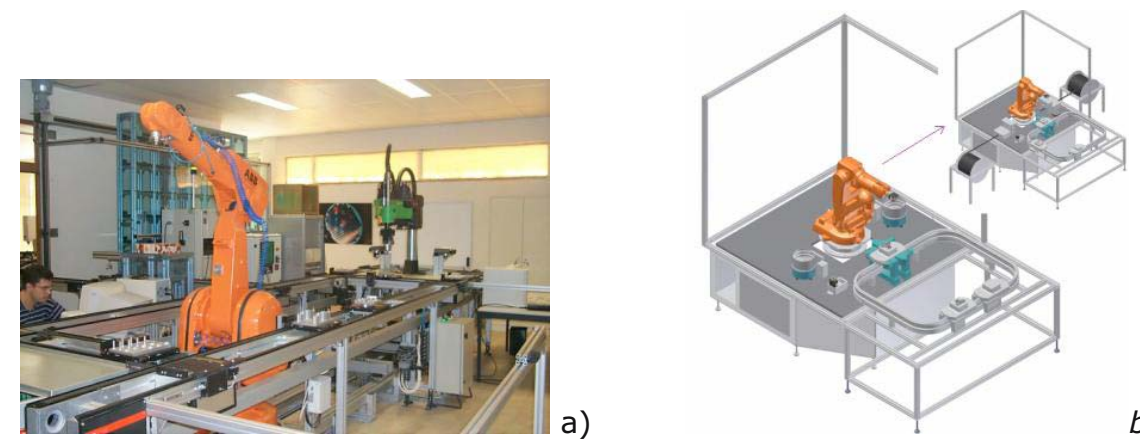

Figure 6. a)The evaluation test case setup; b)Industrial test case setup

\section{CONCLUSIONS}

At present the EAS paradigm is only just starting to take a practical form, and the control solution, ontologies, and methodologies only partially describe the most recent developments. These ideas are now being put into a real industrial scenario through the participation of Electrolux Home Products Italy SpA and UNINOVA. The layout given in Figure $7 \mathrm{~b}$ is being setup for two industrial products (self-configuring \& reconfiguring).

EAS, as with other similar approaches, offers great opportunities for attaining true agility and cost-effective, stepwise automation. The technologies for achieving this are available and there are several partners willing to partake in this endeavour; however, it is vital to point out that EAS does imply that the manner in which we develop and create projects for the development of assembly systems are radically changed, assuming a more synthesis-based approach. 
The experience of this group is that there exists far too strong a resistance to such change in certain academic and industrial circles, and that the creation of new approaches and even new service sectors, all based on EAS and similar approaches, will have to prove their validity through even more elaborate industrial scenarios. Nevertheless, it must be said that at the end of the day it is not the most efficient or technologically advanced solution that may represent the future way of developing assembly systems, but the one that best paves the way to large-scale exploitation.

\section{REFERENCES}

1. "SMEs in Focus- Main results from the 2002 Observatory of European SMEs", submitted to the Enterprise Directorate-General of the European Commission by KPMG Special Services; Observatory of European SMEs, ISBN 92-894-4878-4.

2. The NEMI Roadmap; NEMI, National Electronics Manufacturing Initiative (USA), http://www.nemi.org/Roadmapping/index.html.

3. "Strategic Research Agenda-assuring the future of manufacturing in Europe"; Manufuture Platform-Executive Summary, Dec. 2005, EC.

4. "MANVIS, Manufacturing Visions-Integrating Diverse Perspectives into Pan-European Foresight"; FP6 Support Action, NMP2-CT-2003-507139.

5. "The Future of Manufacturing in Europe 2015-2020-The Challenge for Sustaninability"; FutMan, Institute for Prospective Technological Studies, European Commission Joint Research Centre, EUR 20705 EN.

6. "First Draft Roadmap-deliverable 1.5b"; Project Report-Public, Document 1.5b, EUPASSEvolvable Ultra Precision Assembly, NMP-2-CT-2004-507978; October 2005.

7. "Plants prospering from climate change"; Environment News Services; Pegg,J.R.; International daily Newswire, Washington DC, June 6, 2003.

8. Viewpoint; Onori, M. Assembly Automation Journal 2002b; 22: 8-12.

9. Barata, J., Onori, M. and Frei, R. In ISIE'06 - IEEE International Symposium on Industrial ElectronicsIEEE, Montreal - Canada, 2006b.

10. Ueda, K. Journal of Artificial Intelligence in Engineering 2001; 15: 319-320.

11. Semere, D., Barata, J. Onori, M. Evolvable Systems: Developments and Advance., ISAM 2007 IEEE conference, Ann Arbor, Michigan, USA. July 2007.

12. Holland, J. H. Emergence - From Chaos to Order, Oxford University Press, Oxford, 1998.

13. Johnson, S. Emergence, Penguin group, London, 2001.

14. Barata de Oliveira, J.A.;" Coalition Based Approach for Shop Floor Agility- A multi-Agent Approach"; PhD thesis, Universidade Nova de Lisboa, January 2004 